Document downloaded from:

http://hdl.handle.net/10251/120664

This paper must be cited as:

Garro, E.; Gimenez Gandia, JJ.; Klenner, P.; Gomez-Barquero, D. (2018). InformationTheoretic Analysis and Performance Evaluation of Optimal Demappers for Multi-Layer Broadcast Systems. IEEE Transactions on Broadcasting. 64(4):781-790.

https://doi.org/10.1109/TBC.2018.2799300

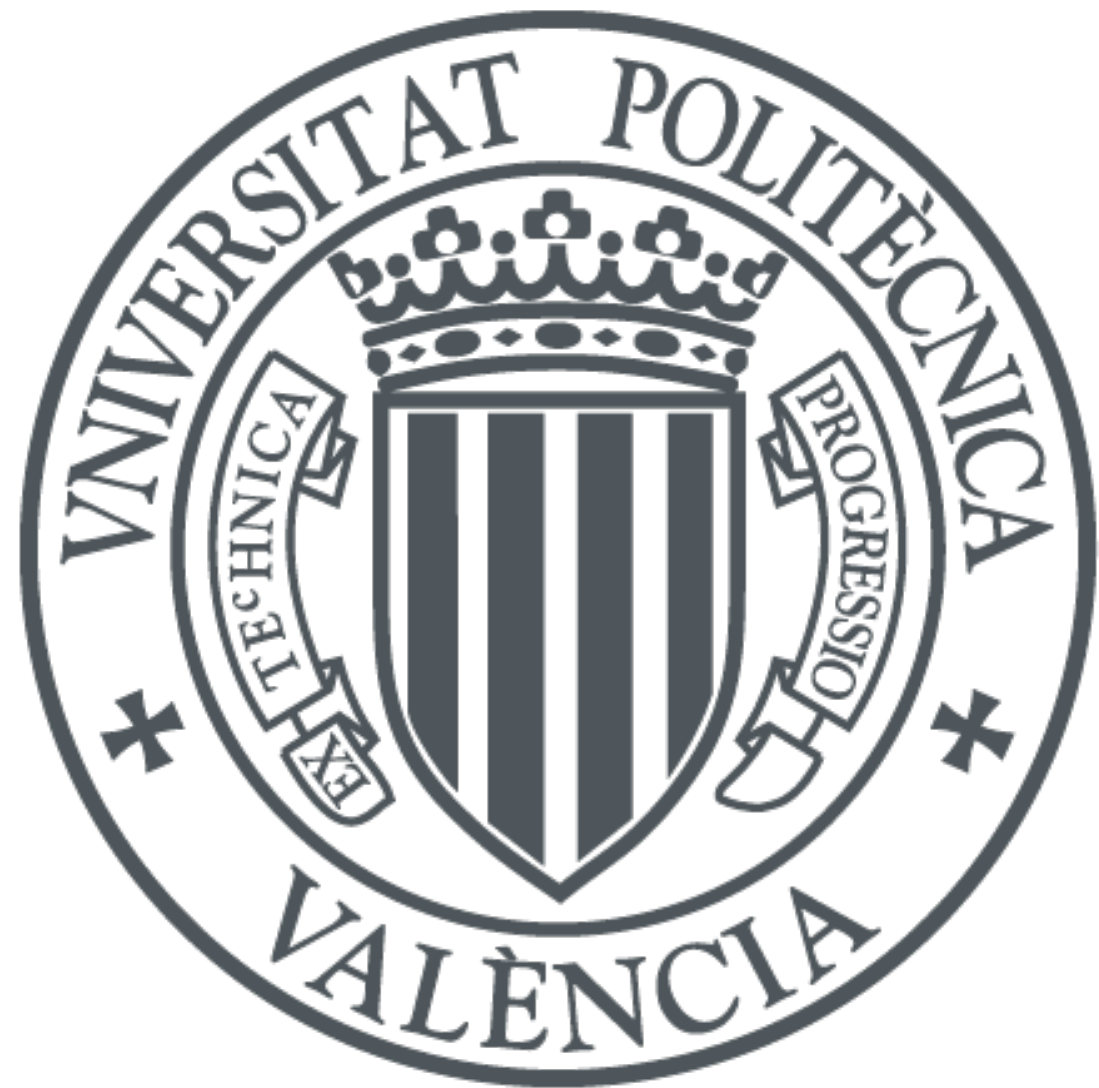

The final publication is available at

http://doi.org/10.1109/TBC.2018.2799300

Copyright Institute of Electrical and Electronics Engineers

Additional Information

(c) 2018 IEEE. Personal use of this material is permitted. Permission from IEEE must be obtained for all other users, including reprinting/ republishing this 


\title{
Information-Theoretic Analysis and Performance Evaluation of Optimal Demappers for Multi-layer Broadcast Systems
}

\author{
Eduardo Garro, Jordi Joan Gimenez, Peter Klenner and David Gomez-Barquero
}

\begin{abstract}
Multi-layer broadcast systems distribute services across time and frequency domain by means of power-division multiplexing. Successive interference cancellation is required, in general, in order to extract the content of all services. For a lowcomplexity implementation, the receiver can obtain the strongest (top-layer) signal assuming underlying signals to behave like thermal noise. The thermal noise assumption may not be valid under certain conditions and a more accurate characterization of the interference could bring improved performance. This paper analyzes the validity of the noise-like assumption considering the power ratio between signals and the required Carrier-to-Noise ratio (CNR) for error-free reception. The main contribution of the paper is the proposal of a demapping algorithm that exploits the knowledge of the constellation of underlying signals. Generalized Mutual Information, performance evaluation, and complexity analysis are provided with the AWGN-like assumptions and with the proposed alternative in order to assess the potential performance improvements that can be achieved.
\end{abstract}

Index Terms-DTT, ATSC 3.0, Layered Division Multiplexing (LDM), WiB, NOMA, LLR demapping

\section{INTRODUCTION}

$\mathbf{M}$ ULTI-layer transmission has been raised as a relevant broadcast technology where the multiplexing of services is performed in the power domain while using $100 \%$ of the frequency and time resources. Implemented as Layered Division Multiplexing (LDM) in ATSC 3.0 [1], the signal consists of the superposition of two signals/layers with different power levels. Each layer, namely Core Layer (CL) and Enhanced Layer (EL), passes through a different Bit-Interleaved Coded Modulation (BICM) chain. This brings the possibility to assign different robustness/capacity characteristics to different services, and hence, to target different reception conditions simultaneously. Once encoded, the signals are aggregated with different power levels.

The concept behind multi-layer transmission has also been considered to allow for frequency reuse-1 networks with Cloud Transmission $[2 \mid$ and $\mathrm{WiB}$ (Wideband reuse-1) |3]. In the WiB concept, all stations are assumed to transmit signals configured with a robust MODCOD (Modulation and Code Rate)

This work was supported by the Ministerio de Educación y Ciencia, Spain (TEC2014-56483-R), co-funded by European FEDER funds. (Corresponding author: Eduardo Garro.)

E. Garro, J.J. Gimenez and D. Gomez-Barquero are with the Universitat Politecnica de Valencia, 46022, Valencia, Spain (e-mail: \{edgarcre,jorgigan,dagobar\}@iteam.upv.es).

P. Klenner is with Panasonic Langen Development Centre, Standardisation Division, Panasonic Europe Ltd., 63225, Langen, Germany (e-mail: peter.klenner@eu.panasonic.com).
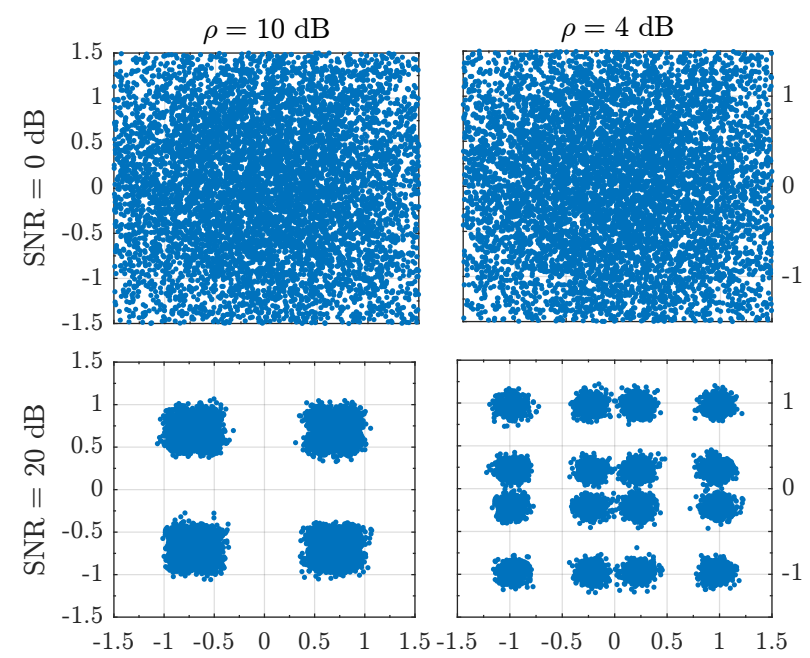

Figure 1. QPSK + QPSK signal with $\rho=10 \mathrm{~dB}$ (left) and $\rho=4 \mathrm{~dB}$ (right). SNR $=0 \mathrm{~dB}$ (top) and $\mathrm{SNR}=20 \mathrm{~dB}$ (bottom).

that would enable reception in a highly interference-limited situation. The received signal consists of the superposition of multiple signals of the same nature (like in LDM) with a different power level according to propagation conditions and transmit power.

Receivers are able to perform the demodulation of the toplayer signal as soon as the received Signal-to-Interferenceplus-Noise ratio (SINR) is larger than the operating Signalto-Noise ratio (SNR) of the selected MODCOD. The other signals/layers can be demodulated by Successive Interference Cancellation (SIC) algorithms. In case of two signals, the power ratio between them can be modeled by means of an Injection Level $(\rho)$.

The commonly used demapping approach is to consider that signals/layers below the target one can be regarded as AWGN-like (Additive White Gaussian Noise) interference [4], [5]. However, potential gains can still be achieved if underlying layers are not considered AWGN. Figure 1 illustrates the impact of $\rho$ and SNR conditions for a multi-layer signal constituted of two QPSK constellations. Top figures show the received constellation symbols in a low SNR region $(0 \mathrm{~dB})$, where AWGN dominates regardless of $\rho$. Lower figures show the symbols in a higher SNR region $(20 \mathrm{~dB})$. It can be noticed from lower figures that for $\rho=10 \mathrm{~dB}$, the QPSK symbols look like being affected by AWGN distribution. On the other hand, with $\rho=4 \mathrm{~dB}$, this assumption is not valid. The resulting 
constellation presents symbols that are a combination of the symbols of the different layers, each one affected by AWGN. Hence, potential performance gains may be achieved if this effect is considered.

This paper expands the initial studies in $|6|$ of a demapping approach for LDM systems in which the AWGN-like interference assumption may not be valid. This new demapping approach considers the distribution of the symbols of the underlying LDM layer when demapping the top-layer signal, which brings a potential gain under certain circumstances at the expense of additional complexity. In addition to $|6|$, the paper evaluates the new demapping concept from a generic point of view, via information theory, and studies the complexity of its implementation at receivers. Furthermore, a new algorithm is proposed, which forwards the a-priori information obtained by the demapping of the top-layer signal to the lowerlayer signal. The implementation of such algorithm may allow eliminating the need of the traditional cancellation process performed in multi-layer systems.

The rest of the paper is structured as follows: Section II presents the formulation of the proposed demapping algorithms, as well as a complexity analysis in terms of number of operations. Section III evaluates and compares the Generalized Mutual Information (GMI) limits of the new approach with the AWGN assumption. Top-layer signal performance results for a wide range of MODCODs and $\rho$ values are shown and discussed in Section IV. Moreover, performance evaluation for the lower-layer signal is also studied. Finally, conclusions are drawn in Section $\mathrm{V}$

\section{Constellation Demapper Alternatives in MULTI-LAYER SYSTEMS}

The received signal, considering that the transmitted signal is composed of two signals/layers, can be modeled by the following expression:

$$
y=x \cdot h+w=\left(\tau \cdot x_{t}+\beta \cdot x_{b}\right) \cdot h+w
$$

where $x_{t}$ and $x_{b}$ denote the top-layer and bottom-layer transmitted complex-valued symbols, with $\tau=1 / \sqrt{1+g^{2}}$ and $\beta=g / \sqrt{1+g^{2}}$ amplitudes, respectively. $g=10^{-\frac{\rho}{20}}$ is the injection level expressed in linear units.

Considering that $x_{t}$ is the first signal to be demodulated, a straightforward approach is to consider $x_{b}$ as an interfering contribution, which can be regarded as AWGN (in the following, Gaussian Demapping) [7]. Alternatively, the Optimum Demapping approach considers $x_{b}$ as useful information by considering its symbol alphabet. Although the demodulation of the bottom-layer signal can be performed by a hard-interference cancellation of the reconstructed top-layer symbols [7], the new method can also be extended to allow for a soft-cancellation approach.

\section{A. Gaussian Approach Demapping (GD)}

1) Top-layer signal $\left(x_{t}\right)$ demapping: The GD assumes the bottom-layer signal $\left(x_{b}\right)$ as additional source of AWGN (with zero mean and single-sided variance $\sigma_{g}^{2}=\beta^{2}$ ).
Using soft-decision decoding, the Log-Likelihood Ratio (LLR), $\Lambda_{t}^{G D}\left(b_{i}\right)$, for each coded bit $b_{i}, i=1, \ldots, m$ is calculated as:

$$
\Lambda_{t}^{G D}\left(b_{i}\right) \triangleq \log \frac{p\left(b_{i}=1 \mid y, h\right)}{p\left(b_{i}=0 \mid y, h\right)}=\log \frac{\sum_{x_{t} \in \zeta_{i}^{1}} p\left(y \mid \tau x_{t}, h\right)}{\sum_{x_{t} \in \zeta_{i}^{0}} p\left(y \mid \tau x_{t}, h\right)}
$$

where $\zeta_{i}^{b}$ denotes the signal subset of constellation points of $x_{t}$ with the $i$-th bit being $b_{i} \in\{0,1\} \cdot p\left(y \mid \tau x_{t}, h\right)$ is the conditional PDF [8] corresponding to the expression:

$$
p\left(y \mid \tau x_{t}, h\right)=\frac{1}{\pi\left(\sigma_{g}^{2}+\sigma_{\omega}^{2}\right)} \exp \left(-\frac{\left|y-h \tau x_{t}\right|^{2}}{\sigma_{g}^{2}+\sigma_{\omega}^{2}}\right)
$$

The obtained LLRs passed through the LDPC decoder so that the estimated bits of the transmitted signal are obtained.

2) Bottom-layer signal $\left(x_{b}\right)$ demapping: To retrieve the bottom-layer signal, the $\Lambda_{t}^{G D}\left(b_{i}\right)$ are LDPC-decoded, remodulated and subtracted from the received signal $y$ of (1). This process is defined as Hard-Cancellation (HC) method. The system model at this point is:

$$
\tilde{y}=y-\tau \hat{x}_{t} \cdot h=\beta \cdot x_{b} \cdot h+w
$$

where $\hat{x}_{t}$ are the re-encoded complex-valued symbols of the top-layer signal.

The LLRs of the bottom-layer signal $\Lambda_{b}^{G D}\left(b_{j}\right)$ for each coded bit $b_{j}$ are finally calculated as:

$$
\Lambda_{b}^{G D}\left(b_{j}\right)=\log \frac{\sum_{x_{b} \in \zeta_{j}^{1}} p\left(\tilde{y} \mid \beta x_{b}, h\right)}{\sum_{x_{b} \in \zeta_{j}^{0}} p\left(\tilde{y} \mid \beta x_{b}, h\right)}
$$

being the PDF $p\left(\tilde{y} \mid \beta x_{b}, h\right)$ defined as:

$$
p\left(\tilde{y} \mid \beta x_{b}, h\right)=\frac{1}{\pi \sigma_{\omega}^{2}} \exp \left(-\frac{\left|\tilde{y}-h \beta x_{b}\right|^{2}}{\sigma_{\omega}^{2}}\right)
$$

\section{B. Optimum Demapping (OD) Approach}

As depicted in Figure 1, the AWGN-like assumption of $x_{b}$ on the $x_{t}$ demodulation may not be accurate in certain conditions. This mismatched assumption can lead to unexpected performance degradation of $x_{t}$. Furthermore, the potential bad estimation of $x_{t}$ could be forwarded to $x_{b}$, since the latter demodulation makes use of the former. Therefore, a new approach that increases $x_{t}$ and $x_{b}$ performances becomes imperative. This section extends the formulation presented in [6] by providing the complete LLR and PDF equations for the implementation of the OD approach for the top-layer signal. For the bottom-layer signal, a soft-cancellation (SC) demapping formulation is also provided considering a-priori LLR values from the top-layer signal.

1) Top-layer signal $\left(x_{t}\right)$ demapping: The proposed Optimum Demapping algorithm assumes the knowledge of the bottom-layer signal constellation $\left(x_{b}\right)$ when demapping the top-layer signal $\left(x_{t}\right)$. To do so, Euclidean distances for all combinations resulting from the sum of the constellations of the two layers are calculated. Assuming the received signal 
of (1), the top-layer signal LLR $\Lambda_{t}^{O D}\left(b_{i}\right)$ is calculated according to:

$$
\Lambda_{t}^{O D}\left(b_{i}\right)=\log \frac{\sum_{x_{t} \in \zeta_{i}^{1}} \sum_{x_{b}} p\left(y \mid \tau x_{t}, \beta x_{b}, h\right)}{\sum_{x_{t} \in \zeta_{i}^{0}} \sum_{x_{b}} p\left(y \mid \tau x_{t}, \beta x_{b}, h\right)}
$$

The second summation term involves all possible transmitted $x_{b}$ values for each transmitted $x_{t}$. The PDF $p\left(y \mid \tau x_{t}, \beta x_{b}, h\right)$ is modeled as:

$$
p\left(y \mid \tau x_{t}, \beta x_{b}, h\right)=\frac{1}{\pi \sigma_{\omega}^{2}} \exp \left(-\frac{\left|y-h\left(\tau x_{t}+\beta x_{b}\right)\right|^{2}}{\sigma_{\omega}^{2}}\right)
$$

2) Bottom-layer signal $\left(x_{b}\right)$ demapping: The same toplayer signal hard-cancellation (HC) process as in Section II-A2 can be performed using the optimum LLR values estimated in (7). However, the top-layer signal remodulation and hardcancellation processes may be omitted if $\Lambda_{t}^{O D}\left(b_{i}\right)$ is regarded as a-priori LLR values on a soft-cancellation (SC) demapping of the bottom-layer signal.

The use of a-priori LLR values in iterative processing is a well-established topic in the field. For example, a similar algorithm was proposed in [9] in the context of iterative demapping for multilevel modulation. It has not been addressed extensively though with regards to broadcasting by means of superposition modulation, where the de-facto standard is set by hard-successive interference cancellation (cf. |7]).

The expression for obtaining the bottom-layer signal LLR $\Lambda_{b}^{O D}\left(b_{j}\right)$ can be written as:

$$
\Lambda_{b}^{O D}\left(b_{j}\right)=\log \frac{\sum_{x_{b} \in \zeta_{j}^{1}} \sum_{x_{t}} p\left(y \mid \tau x_{t}, \beta x_{b}, h\right) P\left(x_{t}\right)}{\sum_{x_{b} \in \zeta_{j}^{0}} \sum_{x_{t}} p\left(y \mid \tau x_{t}, \beta x_{b}, h\right) P\left(x_{t}\right)}
$$

It can be observed that same conditional PDF as (8) is used. However, since bottom-layer signal LLR $\Lambda_{b}^{O D}\left(b_{j}\right)$ are calculated now, all possible transmitted $x_{t}$ values are considered for each transmitted $x_{b} . P\left(x_{t}\right)$, which refers to the transmitted vector probability, can be developed as:

$$
P\left(x_{t}\right)=\prod_{i=1}^{m} P\left(b_{i}\right) \propto \prod_{i=1}^{m} \exp \left(b_{i} \Lambda_{t}^{O D}\left(b_{i}\right)\right)
$$

\section{Demappers Complexity}

The OD approach can potentially provide gains in high SNR regions at the expense of increased complexity. The complexity of the two demapping approaches, GD and OD, is computed in terms of required number of Euclidean distances.

The GD approach for the top-layer signal involves the calculation of the distances between the received signal $y$ and all possible transmitted symbols $x_{t}$, resulting in $2^{m_{t}}$ Euclidean distances, with $m_{t}$ the number of transmitted bits per symbol of the top-layer signal. If top-layer signal hard-cancellation is performed, the bottom-layer signal requires the calculation of the Euclidean distances between the cancelled $\tilde{y}$ and all possible transmitted symbols $x_{b}$, leading to $2^{m_{b}}$, where $m_{b}$ is the number of transmitted bits per symbol of the bottom-layer
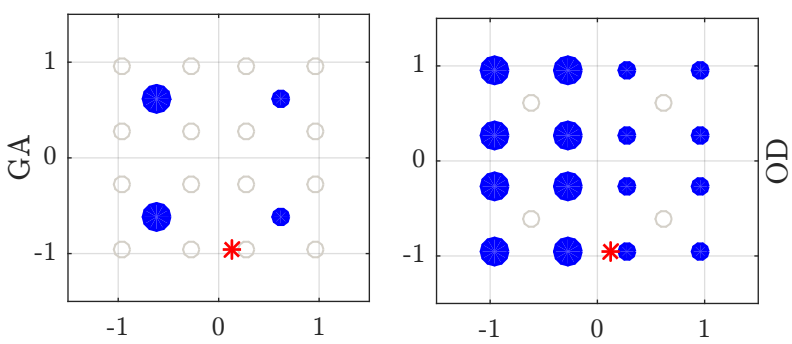

Figure 2. Transmitted points of the constellation (blue) for obtaining the LLR of the received symbol (red) with GD (left), and OD (right) approaches. Thin dots represent the points of $\zeta_{0}^{0}$ while thick dots represent the points of $\zeta_{0}^{1}$.

signal. Therefore, the total number of Euclidean distances to be computed for the two signals is $2^{m_{t}}+2^{m_{b}}$.

On the other hand, the OD demapping approach for the toplayer signal involves the calculation of the distances between the received signal $y$ and all possible $x_{t}$ and $x_{b}$ symbol combinations. This second approach requires $2^{m_{t}+m_{b}}$ Euclidean distances. For the bottom-layer signal, the same number of distances to be computed are needed, but taking into account the a-priori LLR values as in (10). Thus, $2^{m_{t}+m_{b}+1}$ Euclidean distances are needed for the two-signal demodulation with the OD approach.

Figure 2 shows the transmitted symbols that are taken into account for the top-layer signal LLR calculation of the encoded bit $b_{0}$ with the two demapping approaches. For simplicity, a QPSK + QPSK signal is assumed $\left(m_{t}=2\right.$ and $m_{b}=2$ ). Thin dots correspond to points in $\zeta_{0}^{0}$ while thick dots corresponds to points in $\zeta_{0}^{1}$. As it can be observed, the received symbol (asterisk) is closer to one of the OD constellation points (right) than to one of the GD (left). Hence, a better performance can be provided. On the contrary, whereas GD computes $2^{2}=4$ Euclidean distances for getting $\Lambda_{t}^{G D}\left(b_{0}\right)$, OD computes $2^{2+2}=16$ distances for $\Lambda_{t}^{O D}\left(b_{0}\right)$, which can be likened to a 16QAM constellation. In order to reduce the number of Euclidean distances to be computed by OD at the expense of a performance loss, a semi-optimized approach was evaluated in [10|. It was observed that by employing constellation orders lower than the current bottom-layer constellation on the $\Lambda_{t}^{O D}\left(b_{i}\right)$ computation, the performance was degraded at most by $0.4 \mathrm{~dB}$.

Overall, the GD approach provides a low-complexity demapper implementation, which results in the most practical implementation for systems in which the layer demapping results AWGN limited. The OD, with increased demapping complexity, may be appropriate when the layers involved in the demapping process are configured with low order constellations. The demapper based on a-priori LLR values results in the most complex demapper which practical implementation should be carefully evaluated.

\section{INFORMATION-THEORETICAL ANALYSIS OF BICM SYSTEMS}

In order to compare the demapping approaches presented in Section II for the top-layer signal, an information-theoretical study is investigated in terms of the error exponent and 


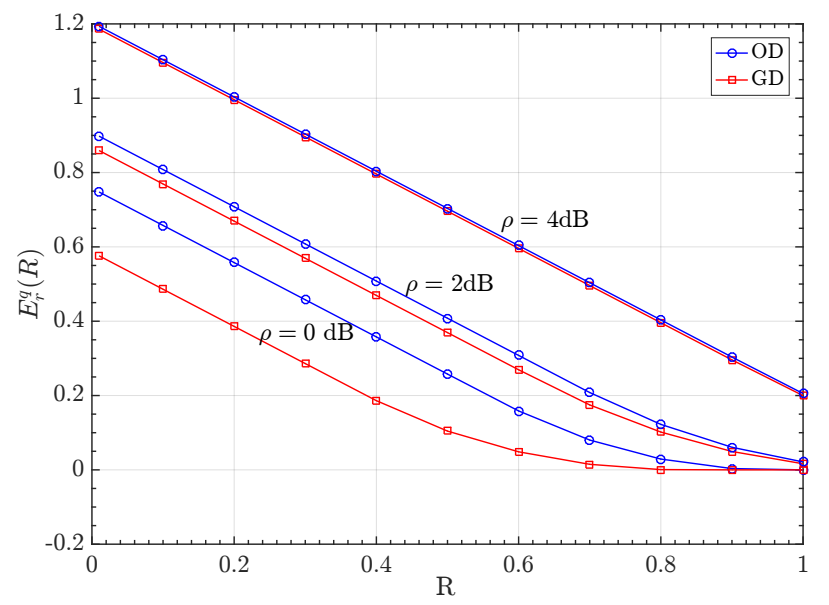

Figure 3. Error exponent for the top layer of QPSK + QPSK with $\rho=0,2$, and $4 \mathrm{~dB}$, and $\mathrm{SNR}=10 \mathrm{~dB}$

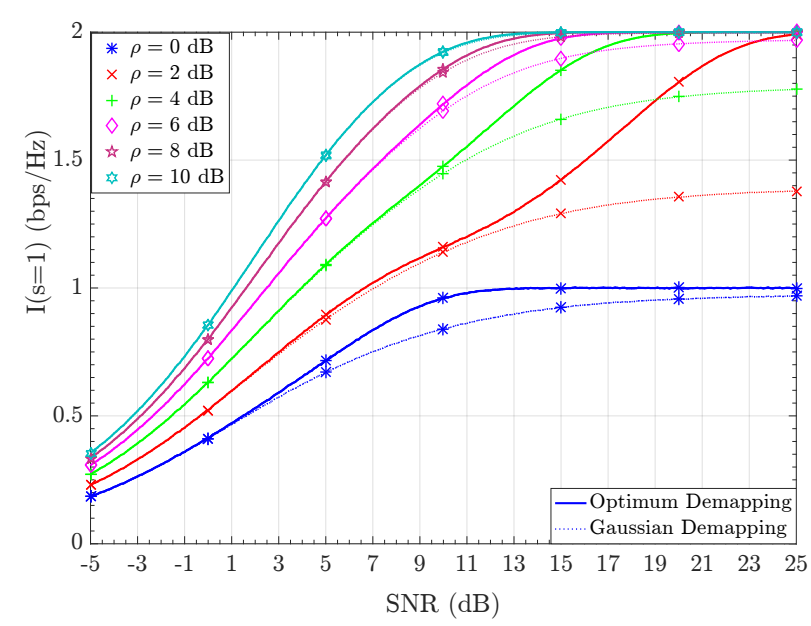

Figure 4. Top-layer signal ( $\left.x_{t}\right)$ QPSK + QPSK I-curves $(s=1)$ for different SNR. $\rho=0,2,4,6,8$, and $10 \mathrm{~dB}$.

Generalized Mutual Information (GMI) of a BICM decoder. Hence, the mismatch effect of GD is also spotlighted.

\section{A. Error Exponent Analysis}

In [11] Gallager derived an upper bound for the average error probability over a random code ensemble and showed that the bound depends on a parameter expediently called error exponent, which in turns depend on Gallager function. Gallager assumed a maximum likelihood decoder with matched PDFs, and showed that the derivative of the Gallager function yields the capacity.

Gallager's derivation can be extended to consider mismatched decoding metrics (see $[12 \mid$ and the references therein). The average error probability over the code ensemble is then denoted by:

$$
\overline{P_{e}} \leq 2^{-N E_{\mathrm{r}}^{q}(R)}
$$

$N$ is the block length, and $E_{\mathrm{r}}^{q}(R)$ is the mismatched random coding error exponent, given by:

$$
E_{\mathrm{r}}^{q}(R)=\max _{0 \leq \varrho<1} \max _{s>0} E_{0}^{q}(\varrho, s)-\varrho R
$$

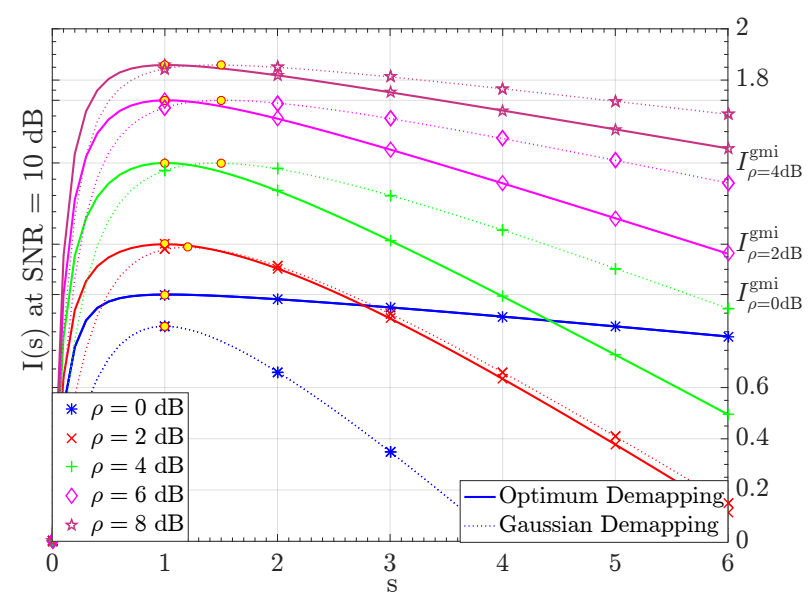

Figure 5. Top-layer signal $\left(x_{t}\right)$ QPSK + QPSK I-curves for different $s$ values at $\mathrm{SNR}=10 \mathrm{~dB} . \rho=0,2,4,6$, and $8 \mathrm{~dB}$.

where $\varrho$ and $s$ are free parameters subject to optimization. $R$ denotes the coding rate.

For the specific case of BICM, the generalized Gallager function $E_{0}^{q}(\varrho, s)$ takes on the form

$$
E_{0}^{\mathrm{bicm}}(\varrho, s)=-\log _{2} \mathbb{E}\left\{\left(\frac{1}{2^{m}} \sum_{x^{\prime}} \prod_{i=1}^{m} \frac{q_{i}\left(b_{i}\left(x^{\prime}\right), Y\right)^{s}}{q_{i}\left(b_{i}(X), Y\right)^{s}}\right)^{\varrho}\right\}
$$

With a slight abuse of notation, the generic decoding metric for the $i$-th bit is given here by

$$
q_{i}\left(b_{i}(x)=b, y\right)=\sum_{x^{\prime} \in \zeta_{b}^{i}} p\left(y \mid x^{\prime}\right)
$$

where the transition probabilities $p\left(y \mid x^{\prime}\right)$ can be based on either matched or mismatched probabilities. The inverse mapping function $b_{i}(x)$ yields the $i$-th bit carried by symbol $x$.

As an example, the error exponent for OD and GD is shown in Figure 3 for top-layer signal employing QPSK with injection levels of $0 \mathrm{~dB}, 2 \mathrm{~dB}$, and $4 \mathrm{~dB}$ at an SNR of $10 \mathrm{~dB}$. The OD yields a larger error exponent over a wide range of code rate $R$ and thus, leads to a more robust system than GD for small injection levels. However, for larger injection levels, e.g., $4 \mathrm{~dB}$, the performance of GD and OD are on par.

\section{B. I-curves}

For any given constellation with spectral efficiency $m_{t}$, the I-curves determine the FEC code rate required to achieve errorfree communication for a particular SNR [13]. The I-curves are obtained as the derivative of the Gallager function:

$$
\begin{aligned}
I(s) & =\left.\frac{d E_{0}^{q}(\varrho, s)}{d \varrho}\right|_{\varrho=0} \\
& =\sum_{i=1}^{m_{t}} \mathbb{E}\left\{\log _{2} \frac{q_{i}(b, y)^{s}}{\frac{1}{2} \sum_{b_{i}=0}^{1} q_{i}\left(b_{i}, y\right)^{s}}\right\}
\end{aligned}
$$

This section computes the I-curves of the top-layer signal bits with GD and OD by Monte Carlo simulations. For such purpose, Equation (15) is expressed in terms of LLRs and 
binary sign function $(\sigma(0)=-1$ and $\sigma(1)=1)$ by substituting $q_{i}\left(b_{i}, y\right)$ for $\exp \left(\frac{1}{2} \sigma\left(b_{i}\right) \Lambda_{t}\left(b_{i}\right)\right)$.

Figure 4 depicts the achievable top-layer signal I-curves for a multi-layer signal constituted by a QPSK + QPSK constellation. GD and OD approaches are considered by using $\Lambda_{t}^{G D}\left(b_{i}\right)$ from (2), and $\Lambda_{t}^{O D}\left(b_{i}\right)$ from (7), respectively. Injection levels $\rho=0,2,4,6,8$, and $10 \mathrm{~dB}$ are evaluated in order to assess their influence in performance. The I-curves are calculated for a range of SNR ${ }^{1}$ values. Note that a matched demapper obtains the GMI at $s=1$ [14]. The results from the figure reveal that both the GD and OD alternatives perform very similar for the SNR region below $10 \mathrm{~dB}$. A clear improvement of the OD is found at higher SNR values. Moreover, for particular $\rho$ values, significant gains can be obtained with OD. Overall, the GD demapper seems to perform very well in noise-limited situations whereas the OD provides an advantage when the interference from the bottom-layer signal dominates. The results obtained via I-curves also reveal that system performance will be limited for certain code rates when using the GD demapper. As an example, for $\rho=2 \mathrm{~dB}$, whereas the OD approach can provide $1.5 \mathrm{bps} / \mathrm{Hz}$ with a degradation of about $10 \mathrm{~dB}$ with respect to the single-layer case $(\rho=10 \mathrm{~dB})$, the GD approach cannot reach error-free reception.

On the other hand, I-curves have also been obtained for a QPSK + 64QAM signal. It was observed that at high SNR regions, the I-curves for the top-layer signal are reduced compared to QPSK + QPSK signal. This behavior refuses the conclusion extracted from [6]. The obtained results are not provided in this section, but performance results are presented and discussed in Section IV

\section{Generalized Mutual Information Analysis}

In [12], the GMI is defined as the supremum of the I-curves relative to $s>0$

$$
I^{\mathrm{gmi}}=\sup _{s>0} I(s)
$$

As it was proved in [14], I-curves with $s=1$ provide $I^{\text {gmi }}$ when a matched PDF is considered. Therefore, the Icurves presented in Figure 4 only represents $I^{\text {gmi }}$ for OD, and an optimization of parameter $s$ may be applied to the GD. Figure 5 illustrates the I-curves with the same configuration and injection levels for $S N R=10 \mathrm{~dB}$ and different $s$ values. As it can be observed, $I^{\text {gmi }}$ is always obtained at $s=1$ for OD, but varies for GD between $s=\{1-1.5\}$. This optimization process of $s$ is not straightforward on real receiver implementations. Reference [12] explains that an optimal LLR scaling in a mismatched decoder (as GD) can increase its performance in the same way as the optimization of $s$. Nevertheless, the implementation of a proper LLR metric scaling is out of the scope of the paper, as it would require of a more sophisticated receiver.

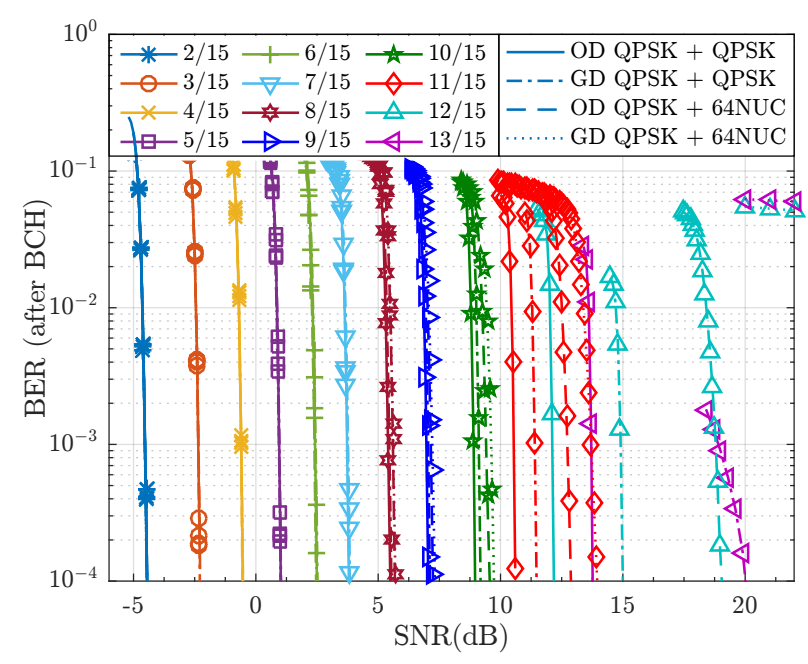

Figure 6. $x_{t}$ BER performance with $\rho=4 \mathrm{~dB} . x_{b}$ QPSK 13/15 and 64NUC $13 / 15$, AWGN channel

Table I

SimULATION SETUP

\begin{tabular}{|c|c|c|c|}
\hline \hline \multicolumn{2}{|c|}{ Top-layer Performance } & \multicolumn{2}{c|}{ Bottom-layer Performance } \\
\hline Parameter & Value & Parameter & Value \\
\hline$x_{t}$ MOD & 2 & $x_{t}$ MOD & 2 \\
\hline$x_{t}$ COD & $2 / 15-13 / 15$ & $x_{t}$ COD & $4 / 15,10 / 15$ \\
\hline$x_{b}$ MOD & $2,4,6,8$ & $x_{b}$ MOD & $2,4,6,8$ \\
\hline$x_{b}$ COD & $13 / 15$ & $x_{b}$ COD & $4 / 15,10 / 15$ \\
\hline$\rho$ (dB) & $0,1,2,3,4,5,6$ & $\rho$ & 2,4 \\
\hline$x_{b}$ Channel Model & $\begin{array}{c}\text { AWGN } \\
\text { i.i.d. Rayleigh }\end{array}$ & $x_{b}$ Channel Model & $\begin{array}{c}\text { AWGN } \\
\text { Rice (DVB-F1) }\end{array}$ \\
\hline \hline
\end{tabular}

\section{Performance Analysis}

Next, the GD and OD approaches are evaluated by considering LDM in an ATSC 3.0 physical layer simulations ${ }^{2}$.

A comparison for the top-layer signal is conducted in Section IV-A and IV-B, and for the bottom-layer signal in Section IV-C . Different MODCODs for the two signals as well as different injection levels $\rho$ have been assumed. An AWGN and an i.i.d. Rayleigh channel model for the top-layer signal are considered. For the bottom-layer signal, an AWGN and a Rice (DVB-F1) channel model are considered. Ideal channel estimation is assumed. Table I presents a summary for the different considered ATSC 3.0 configurations [15].

\section{A. Performance of top-layer signal $\left(x_{t}\right)$ in AWGN channel}

Figure 6 shows the performance of the top-layer signal $x_{t}$ for the different MODCODs under study, when $x_{b}$ is set to either QPSK or 64NUC with $\rho=4 \mathrm{~dB}$. It is shown that for $x_{t}$ coding rates below $7 / 15$, the SNR threshold of the four configurations are the same. Particularly, it can be observed that the SNR thresholds of $4 / 15$ and $6 / 15$ are, -0.5 , and 2.7

\footnotetext{
${ }^{1}$ Under the assumption of normalized transmission symbols $\mathrm{E}\left[|x|^{2}\right]=1$, the SNR is equivalent to the inverse of the noise variance $\mathrm{SNR}=1 / \sigma_{w}^{2}$

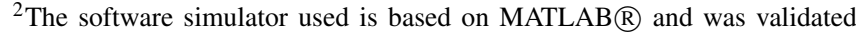
during the standardization process of ATSC 3.0.
} 

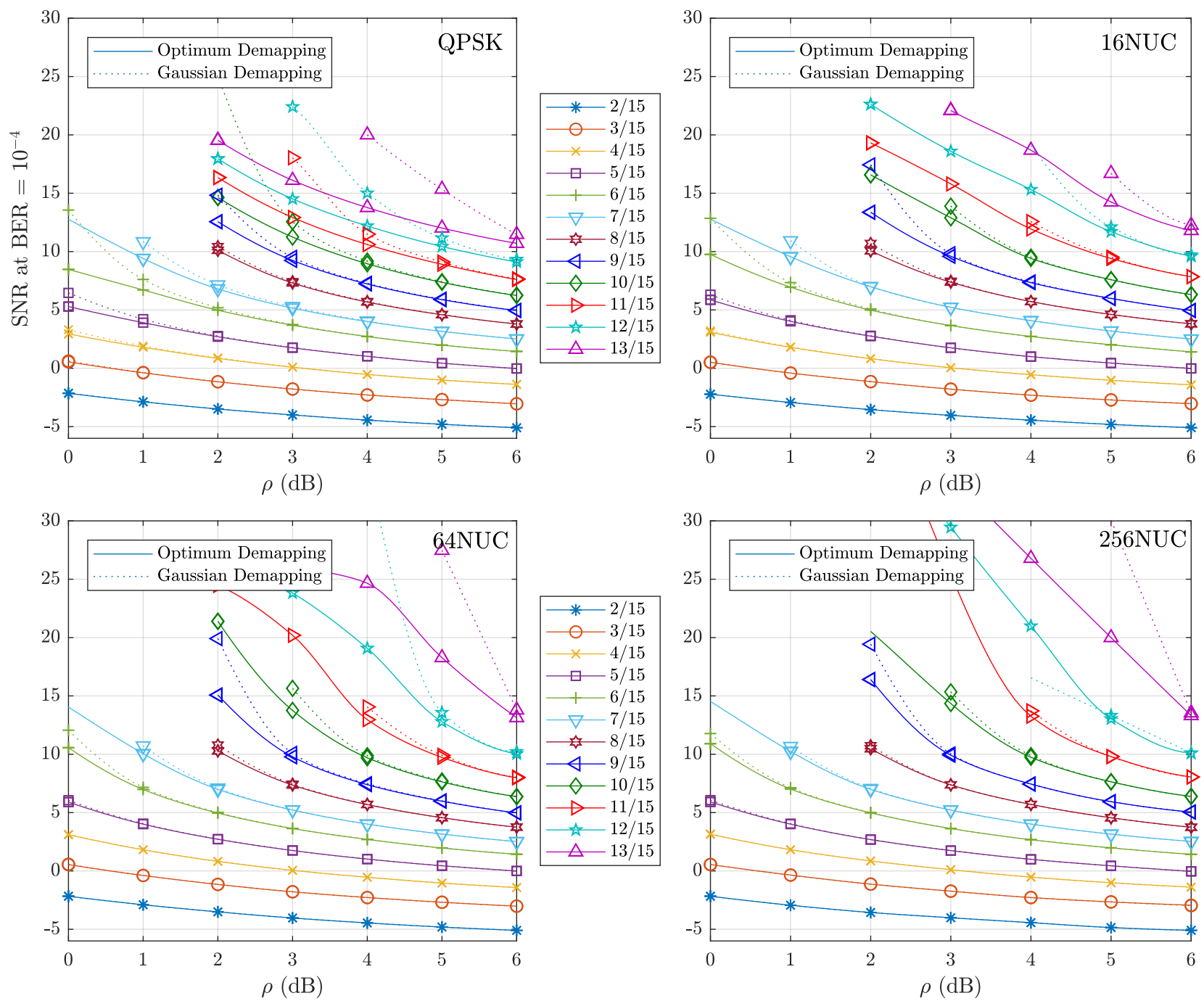

Figure 7. SNR thresholds for all the $x_{t}$ MODCODs and $\rho=1-6 \mathrm{~dB} . x_{b}$ QPSK (top-left), 16NUC (top-right), 64NUC (bottom-left), and 256NUC (bottom-right) in an AWGN channel.

$\mathrm{dB}$, respectively, which fits with the results from [10]. The performance gains of OD are noticeable for SNRs greater than $10 \mathrm{~dB}$, i.e. from $10 / 15 x_{t}$ code rates. This confirms the conclusions in Section III-B. Last, it can also be seen that if $x_{b}$ uses a 64NUC instead of QPSK, there is a performance degradation for $x_{t}$. Moreover, it can be seen that $x_{t} 12 / 15$ and 13/15 cannot achieve error-free reception with the GD demapper if $x_{b}$ a 64NUC constellation.

For a more exhaustive performance study, the SNR thresholds of all $x_{t}, x_{b}$ and $\rho$ values established in Table I are shown in Figure 7 for AWGN channel. In top-left part of the figure, where both signals use same modulation order (as in WiB systems), it can be observed that both demappers provide similar performance for low $\rho$ values at low $x_{t}$ coding rates $(3 / 15-7 / 15)$. However, when the operational SNR is above 5 $\mathrm{dB}$, i.e. when $\rho$ is $1 \mathrm{~dB}$ or smaller, OD outperforming arises. Appreciable gains can also be observed at high $x_{t}$ coding rates $(8 / 15$ - 13/15) from $\rho=5 \mathrm{~dB}$. These statements are applicable to the top-right part of the figure as well, where $x_{b}$ is using a 16NUC modulation order. In particular, for QPSK 13/15 and $\rho=5 \mathrm{~dB}$, the SNR can be $3 \mathrm{~dB}$ lower with OD if a QPSK or a 16NUC is assumed for the $x_{b}$. This could also lead to a potential capacity increase for the same $\rho$ and SNR threshold. From the left part, assuming a fixed $\rho=3 \mathrm{~dB}$, whereas maximum $x_{t}$ MODCOD with GD for a $\mathrm{SNR}=13 \mathrm{~dB}$ is QPSK $10 / 15$, OD can increase the capacity allowing the use of QPSK 11/15.

The bottom part of Figure 7 modifies the lower-layer signal constellation to a 64NUC or 256NUC (commonly used in ATSC 3.0 LDM studies). In these cases, compared to top part, it can be seen an $x_{t}$ performance degradation in high SNR regions for both demapping algorithms. Taking previous configuration, for QPSK $13 / 15$ and $\rho=5 \mathrm{~dB}$, the $x_{t}$ SNR can be $9 \mathrm{~dB}$ lower with OD if a 64NUC is assumed for the $x_{b}$. Furthermore, if a 256NUC is assumed for the $x_{b}$ with $\rho=5 \mathrm{~dB}$, Quasi-Error-Free (QEF) conditions cannot be achieved for GD. This demonstrates that the top-layer signal performance depends on the lower-layer signal constellation when the power ratios and $x_{t}$ coding rates are in the critical region. In $|6|$, the impact of the $x_{b}$ constellation onto $x_{t}$ 
Table II

OD GAINS (DB) FOR QPSK + QPSK / QPSK + 64NUC IN AWGN CHANNEL

\begin{tabular}{|c|ccccccc|}
\hline \hline & \multicolumn{7}{|c|}{$\rho(\mathrm{dB})$} \\
\hline$x_{t}$ & $\mathbf{0}$ & $\mathbf{1}$ & $\mathbf{2}$ & $\mathbf{3}$ & $\mathbf{4}$ & $\mathbf{5}$ & $\mathbf{6}$ \\
$\mathbf{2 / 1 5}$ & $0,0 / 0,0$ & $0,0 / 0,0$ & $0,0 / 0,0$ & $0,0 / 0,0$ & $0,0 / 0,0$ & $0,0 / 0,0$ & $0,0 / 0,0$ \\
$\mathbf{3 / 1 5}$ & $0,0 / 0,0$ & $0,0 / 0,0$ & $0,0 / 0,0$ & $0,0 / 0,0$ & $0,0 / 0,0$ & $0,0 / 0,0$ & $0,0 / 0,0$ \\
$\mathbf{4 / 1 5}$ & $0,4 / 0,0$ & $0,1 / 0,0$ & $0,0 / 0,0$ & $0,0 / 0,0$ & $0,0 / 0,0$ & $0,0 / 0,0$ & $0,0 / 0,0$ \\
$\mathbf{5 / 1 5}$ & $1,2 / 0,2$ & $0,3 / 0,0$ & $0,0 / 0,0$ & $0,0 / 0,0$ & $0,0 / 0,0$ & $0,0 / 0,0$ & $0,0 / 0,0$ \\
$\mathbf{6 / 1 5}$ & $5,1 / 1,5$ & $0,9 / 0,2$ & $0,2 / 0,0$ & $0,0 / 0,0$ & $0,0 / 0,0$ & $0,0 / 0,0$ & $0,0 / 0,0$ \\
$\mathbf{7 / 1 5}$ & $-/-$ & $1,4 / 0,7$ & $0,3 / 0,1$ & $0,1 / 0,0$ & $0,0 / 0,0$ & $0,0 / 0,0$ & $0,0 / 0,0$ \\
$\mathbf{8} \mathbf{1 5}$ & $-/-$ & $-/-$ & $0,3 / 0,5$ & $0,1 / 0,0$ & $0,0 / 0,0$ & $0,0 / 0,0$ & $0,0 / 0,0$ \\
$\mathbf{9 / 1 5}$ & $-/-$ & $-/-$ & $2,3 / 4,8$ & $0,3 / 0,3$ & $0,1 / 0,0$ & $0,0 / 0,0$ & $0,0 / 0,0$ \\
$\mathbf{1 0 / 1 5}$ & $-/-$ & $-/-$ & $10,4 /-$ & $1,3 / 1,9$ & $0,2 / 0,2$ & $0,0 / 0,0$ & $0,0 / 0,0$ \\
$\mathbf{1 1 / 1 5}$ & $-/-$ & $-/-$ & $7,4 /-$ & $5,1 /-$ & $0,9 / 1,1$ & $0,2 / 0,2$ & $0,0 / 0,0$ \\
$\mathbf{1 2 / 1 5}$ & $-/-$ & $-/-$ & $8,1 /-$ & $7,9 /-$ & $2,8 / 15,9$ & $0,7 / 0,8$ & $0,2 / 0,2$ \\
$\mathbf{1 3 / 1 5}$ & $-/-$ & $-/-$ & $-/-$ & $-/-$ & $6,2 /-$ & $3,3 / 9,2$ & $0,8 / 0,7$ \\
\hline \hline
\end{tabular}

performance was only observed at practical regions for LDM operation, so that the system was only AWGN limited. The OD gains with respect to GD when either a QPSK or a 64NUC is used for the $x_{b}$ are summarized in Table II

\section{B. Performance of top-layer signal $\left(x_{t}\right)$ in i.i.d. Rayleigh channel}

In order to assess the same study in a more realistic scenario, an i.i.d. Rayleigh fading channel modeling portable reception is assumed. Figure 8 presents the SNR thresholds for this channel and Table III summarizes the OD gains when $x_{b}$ uses a QPSK or a 64NUC.

A general performance degradation can be observed in all configurations because of the more challenging conditions of this channel. From top figures, it can be seen that low $x_{t}$ coding rates $(3 / 15$ - 7/15) perform similarly for both demapping algorithms at low $\rho$ values, as it occurred with AWGN channel. However, due to the performance degradation increase in portable reception conditions, performance differences can now be seen at a lower operational SNR. In particular, for the $0 \mathrm{~dB}$ operational SNR of WiB systems, 0.9 $\mathrm{dB}$ gains are obtained by OD for QPSK $2 / 15$ with $\rho=0 \mathrm{~dB}$, $0.4 \mathrm{~dB}$ for QPSK $3 / 15$ with $\rho=2 \mathrm{~dB}$, and $0.1 \mathrm{~dB}$ for QPSK $4 / 15$ with $\rho=5 \mathrm{~dB}$. On the other hand, for high $x_{t}$ coding rates $(8 / 15$ - 11/15), which are out of the WiB discussion, the same trend is followed, and noticeable OD gains are now observed from $\rho=6 \mathrm{~dB}$. Particularly, QPSK 12/15 and 13/15 with $\rho=5 \mathrm{~dB}$ can only achieve QEF with OD.

In another vein, the top-layer signal performance dependance on the lower-layer signal constellation is confirmed for a portable reception scenario when top figures are compared with bottom ones. Furthermore, if $x_{b}$ is constituted by a $64 \mathrm{NUC}$ or a $256 \mathrm{NUC}, x_{t}$ performance degradation is observed for both demapping algorithms at high SNR regions, but also for GD at low SNR regions.

\section{Performance of bottom-layer signal $x_{b}$}

The $x_{b}$ performance taking into account the softcancellation by the a-priori $x_{t}$ LLRs (SC) and the traditional
Table III

OD GAINS (DB) FOR QPSK + QPSK / QPSK + 64NUC IN I.I.D. RAYLEIGH CHANNEL

\begin{tabular}{|c|ccccccc|}
\hline \hline & \multicolumn{7}{|c|}{$\rho(\mathrm{dB})$} \\
\hline$x_{t}$ & $\mathbf{0}$ & $\mathbf{1}$ & $\mathbf{2}$ & $\mathbf{3}$ & $\mathbf{4}$ & $\mathbf{5}$ & $\mathbf{6}$ \\
$\mathbf{2 / 1 5}$ & $0,9 / 0,4$ & 0,4 & 0,2 & $0,1 / 0,0$ & $0,0 / 0,0$ & $0,0 / 0,0$ & $0,0 / 0,0$ \\
$\mathbf{3 / 1 5}$ & $1,8 / 1,3$ & $0,7 / 0,6$ & $0,4 / 0,4$ & $0,2 / 0,3$ & $0,0 / 0,2$ & $0,0 / 0,0$ & $0,0 / 0,0$ \\
$\mathbf{4 / 1 5}$ & $3,4 / 4,9$ & $1,4 / 1,7$ & $0,6 / 0,8$ & $0,4 / 0,4$ & $0,2 / 0,3$ & $0,1 / 0,1$ & $0,0 / 0,0$ \\
$\mathbf{5 / 1 5}$ & $12,9 /-$ & $2 / 5,2$ & $0,8 / 1,5$ & $0,4 / 0,7$ & $0,3 / 0,3$ & $0,1 / 0,1$ & $0,0 / 0,0$ \\
$\mathbf{6 / 1 5}$ & $-/-$ & $5,6 /-$ & $1,3 / 3,8$ & $0,6 / 1,3$ & $0,3 / 0,6$ & $0,2 / 0,3$ & $0,0 / 0,1$ \\
$\mathbf{7 / 1 5}$ & $-/-$ & $-/-$ & $2,8 /-$ & $0,9 / 2,8$ & $0,4 / 1,0$ & $0,2 / 0,5$ & $0,1 / 0,2$ \\
$\mathbf{8 / 1 5}$ & $-/-$ & $-/-$ & $-/-$ & $2,5 / 10,8$ & $1,0 / 1,8$ & $0,4 / 0,7$ & $0,2 / 0,3$ \\
$\mathbf{9 / 1 5}$ & $-/-$ & $-/-$ & $-/-$ & $6,7 /-$ & $1,9 / 5$ & $0,8 / 1,5$ & $0,4 / 0,6$ \\
$\mathbf{1 0 / 1 5}$ & $-/-$ & $-/-$ & $-/-$ & $-/-$ & $6,3 /-$ & $2,2 / 3,8$ & $1,1 / 1,3$ \\
$\mathbf{1 1 / 1 5}$ & $-/-$ & $-/-$ & $-/-$ & $-/-$ & $-/-$ & $5,7 /-$ & $2,3 / 3,2$ \\
\hline \hline
\end{tabular}

Table IV

OD AND SC GAINS (DB) FOR AWGN CHANNEL

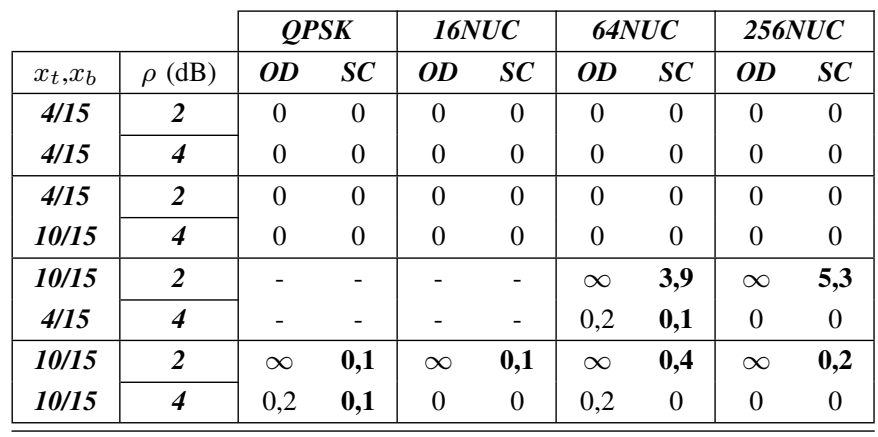

$x_{t}$ hard-cancellation (HC) is evaluated next. To do so, different configurations have been considered: GD for the top-layer plus $\mathrm{HC}$, and OD for the top-layer with both $\mathrm{HC}$ and $\mathrm{SC}$ for the bottom layer. Again, QPSK + QPSK, QPSK + 16NUC, QPSK + 64NUC, and QPSK + 256NUC configurations have been studied. Only $\rho=2$ and $4 \mathrm{~dB}$ are studied, as they represent low and high $\rho$ values, respectively. The $x_{b}$ SNRs at BER $=10^{-4}$ for AWGN channel and DVB-F1 channel are shown in Figure 9, and summarized in Table IV, and Table V respectively. DVB-F1 models a fixed reception channel, which is the potential target of the $x_{b}$ service.

For the WiB study case (QPSK + QPSK) and for

Table V

OD AND SC GAINS (DB) FOR DVB-F1 RICE CHANNEL

\begin{tabular}{|c|c|c|c|c|c|c|c|c|c|}
\hline \multirow[b]{2}{*}{$x_{t}, x_{b}$} & \multirow[b]{2}{*}{$\rho(\mathrm{dB})$} & \multicolumn{2}{|c|}{ QPSK } & \multicolumn{2}{|c|}{$16 N U C$} & \multicolumn{2}{|c|}{ 64NUC } & \multicolumn{2}{|c|}{$256 N U C$} \\
\hline & & $O D$ & $S C$ & $O D$ & $S C$ & $O D$ & $S C$ & $O D$ & $S C$ \\
\hline $4 / 15$ & 2 & 0,2 & 0 & 0 & 0 & 0 & 0 & 0 & 0 \\
\hline $4 / 15$ & 4 & 0 & 0 & 0 & 0 & 0 & 0 & 0 & 0 \\
\hline $4 / 15$ & 2 & 0 & 0 & 0 & 0 & 0 & 0 & 0 & 0 \\
\hline $10 / 15$ & 4 & 0 & 0 & 0 & 0 & 0 & 0 & 0 & 0 \\
\hline $10 / 15$ & 2 & - & - & - & - & $\infty$ & 3,8 & $\infty$ & 5,1 \\
\hline $4 / 15$ & 4 & - & - & - & - & 1 & 0,2 & 0 & 0 \\
\hline $10 / 15$ & 2 & $\infty$ & 0,1 & $\infty$ & 0,1 & $\infty$ & 0,3 & $\infty$ & 0,2 \\
\hline $10 / 15$ & 4 & 0,7 & $\mathbf{0 , 1}$ & 0 & 0 & 0,2 & 0 & 0 & 0 \\
\hline
\end{tabular}



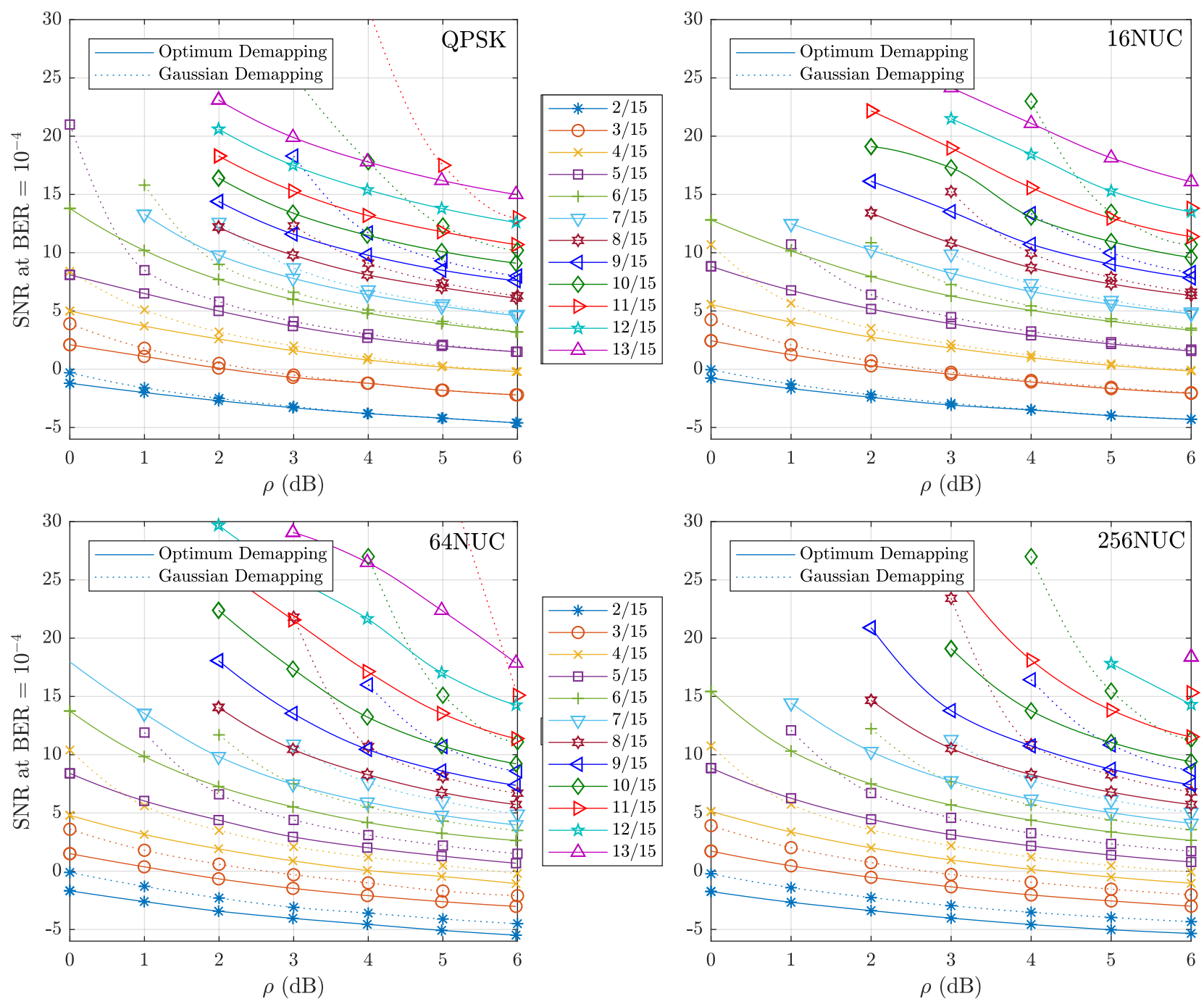

Figure 8. SNR thresholds for all the $x_{t}$ MODCODs and $\rho=1-6 \mathrm{~dB} . x_{b}$ QPSK (top-left), 16NUC (top-right), 64NUC (bottom-left), and 256NUC (bottom-right) in an i.i.d. Rayleigh channel.

QPSK+16NUC configuration, it can be observed that the use of $\mathrm{HC}$ or $\mathrm{SC}$ for the $x_{b}$ signal does not introduce significant gains for a robust $x_{t}$ MODCOD (4/15). The $x_{b}$ SNR threshold is mainly affected by the power reduction due to the injection level $\rho$. Nevertheless, when OD approach provides gains for the $x_{t}$, i.e. at high $x_{t}$ code rate (10/15), the performance of the $x_{b}$ is also improved. This is particularly relevant in the case of $\rho=2 \mathrm{~dB}$ where GD cannot achieve QEF reception (grey bar) for $x_{t}$, and so for $x_{b}$. Regarding SC-HC comparison, it can be observed that SC provides a slight $x_{b}$ improved performance in these conditions $(0,1 \mathrm{~dB})$ for both channels.

For the QPSK + 64NUC, and QPSK+256NUC cases, the differences in $x_{b}$ performance because of the top-layer signal demapping approach (GD vs OD) can be again noticed for the weak $x_{t}$ code rate $10 / 15$, when $\rho=2 \mathrm{~dB}$. On the other hand, the $x_{b}$ performance increase due to the use of SC is now increased. Large gains (about $4 \mathrm{~dB}$ for $64 \mathrm{NUC}$ and 5,3 $\mathrm{dB}$ for $256 \mathrm{NUC}$ ) can be achieved if a robust $x_{b}$ code rate is configured, but also are observed with high $x_{b}$ code rate (around $0,3 \mathrm{~dB}$ for both $x_{b}$ modulation orders) for AWGN channel.

Similar gains are obtained when the more realistic fixedrooftop channel is assumed. From Table V , 3,8 dB gains are obtained by SC when $x_{b}$ is constituted by a $64 \mathrm{NUC} 4 / 15$ and $5,1 \mathrm{~dB}$ when is formed by a $256 \mathrm{NUC} 4 / 15$ ).

\section{CONCLuSions}

This paper studies different demapping approaches for multi-layer broadcast systems from a generic point of view. Underlying signals have commonly been assumed as AWGNlike interference when demapping the top-layer constellation (GD). As previously introduced in [6], this assumption may not be valid when the power of the layers is similar and high code-rates are configured for the top-layer signal.

The paper provides results in terms of error exponent and generalized mutual information by means of Monte-Carlo simulations, covering a wide range of operational points. The performance has been crosschecked with ATSC 3.0 physical layer simulations and compared to the results presented in [6]. The optimum demapping (OD) approach, which considers the 

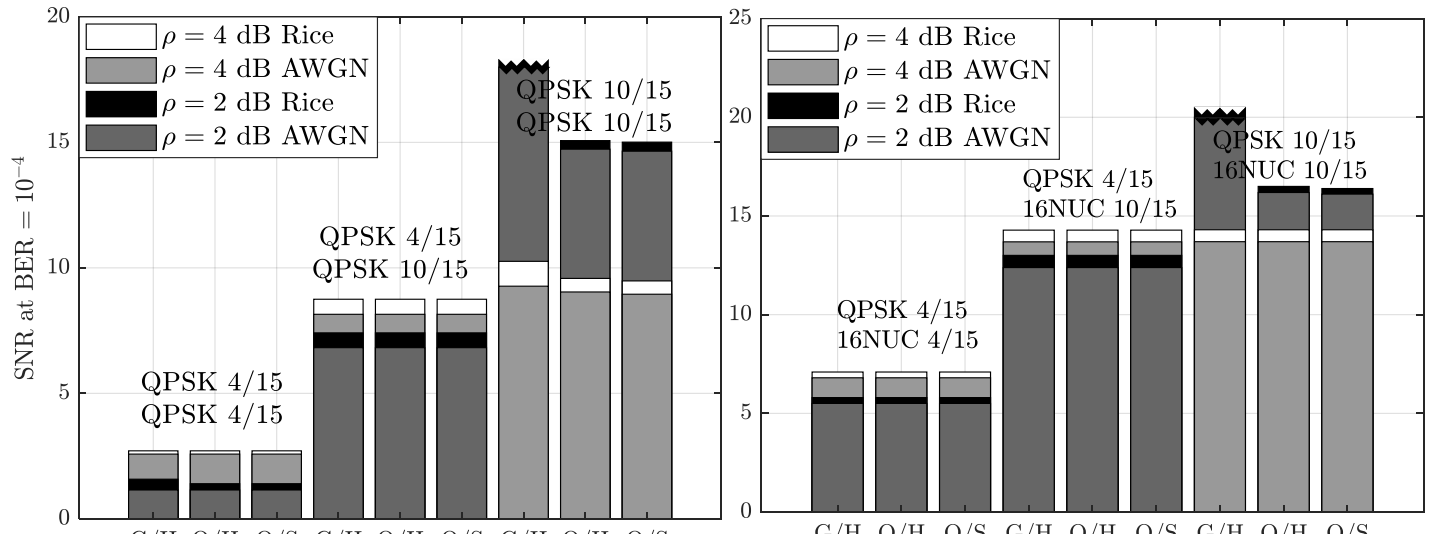

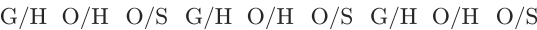

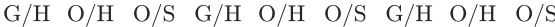

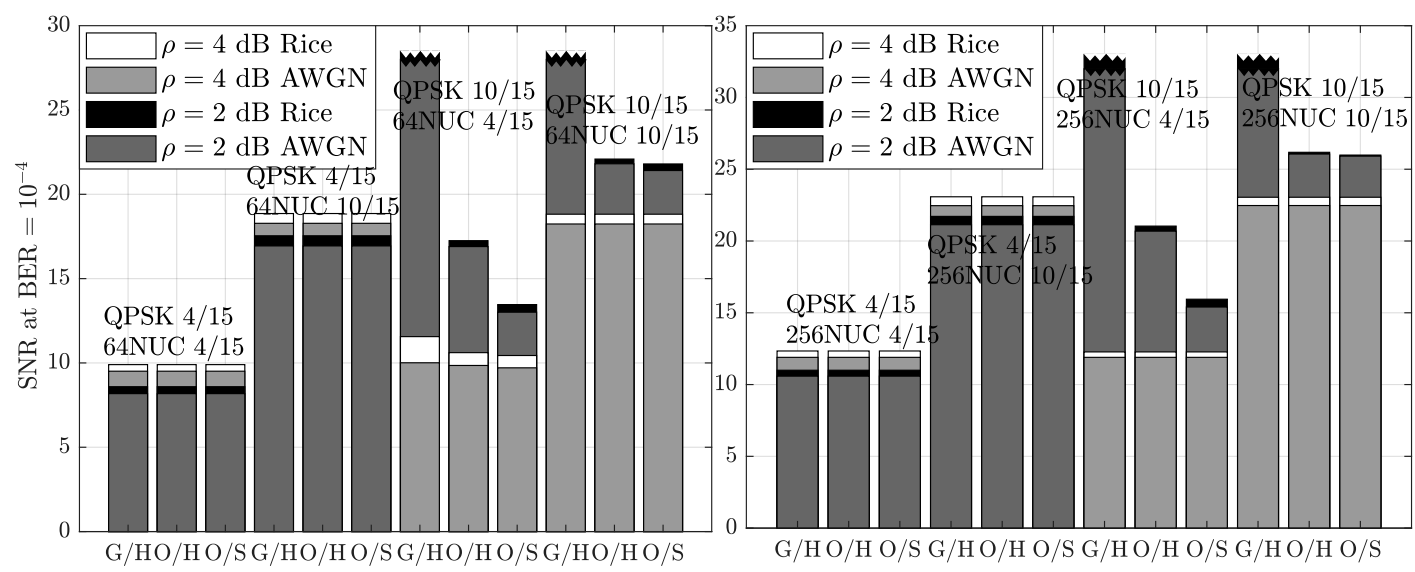

Figure 9. $x_{b}$ SNR thresholds for QPSK + QPSK (top-left), QPSK + 16NUC (top-right), QPSK + 64NUC (bottom-left), and QPSK + 256NUC (bottom-right) with $\rho=2$ and $4 \mathrm{~dB}$ for AWGN and Rice channels (G/H: Gaussian Demapping and Hard-Cancellation, O/H: Optimum Demapping and Hard-Cancellation, O/S: Optimum Demapping and Soft-Cancellation).

knowledge of the symbol alphabet of the underlying constellation brings potential gains at the expense of a complexity increase comparable to a higher modulation order (in terms of Euclidean distances to be computed). It was observed that OD gains depend on the power ratio between layers $(\rho)$, the toplayer code-rate and the lower-layer constellation. They vary from $0 \mathrm{~dB}$ (at high $\rho$, and low top-layer signal code-rate) up to $10 \mathrm{~dB}$ (at low $\rho$, and high top-layer signal code rate). Moreover, the OD method brings a performance increase up to $4 \mathrm{~dB}$ for the underlying layers when a cancellation method based on soft a-priori information transfer (SC) is applied.

The expected gains by the OD demapping may be useful in systems employing robust signals (i.e. QPSK modulation order) with low power differences. Although the operation points in which gains are obtained are less attractive for ATSC 3.0 LDM operation, systems such as WiB can benefit from high gains when e.g. the same QPSK signal is transmitted from multiple stations.

Further studies should consider the performance analysis using other fading channel models, as well as the impact of introducing more than two layers. In addition, since the a-priori information transfer from top to bottom layer signals have been demonstrated to significantly improve performance, an iterative extension, also considering the transfer from bottom to top layer, should be analyzed as well as their implications in terms of complexity. Other implementation aspects, such as the increased power consumption by LDPC decoders in low SNR conditions, can also be considered.

\section{REFERENCES}

[1] L. Fay, L. Michael, D. Gomez-Barquero, N. Ammar, and M. W. Caldwell, "An Overview of the ATSC 3.0 Physical Layer Specification," IEEE Trans. Broadcast., vol. 62, no. 1, March 2016.

[2] Y. Wu, B. Rong, K. Salehian, and G. Gagnon, "Cloud Transmission: A New Spectrum-Reuse Friendly Digital Terrestrial Broadcasting Transmission System," IEEE Trans. Broadcast., vol. 58, no. 3, pp. 329-337, Sept 2012.

[3] E. Stare, J. J. Gimenez, and P. Klenner, "WiB - A New System Concept for Digital Terrestrial Television (DTT)," The Best of IET and IBC 20162017, vol. 8, pp. 4-9, 2016.

[4] S. Gadkari and K. Rose, "Time-Division versus Superposition Coded Modulation Schemes for Unequal Error Protection," IEEE Trans.on Communications, vol. 47, no. 3, pp. 370-379, Mar 1999.

[5] L. Zhang et al., "Layered Division Multiplexing: Theory and Practice," IEEE Trans. Broadcast., vol. 62, no. 1, March 2016.

[6] C. Regueiro et al., "LLR Reliability Improvement for Multilayer Signals," IEEE Trans. on Broadcast., vol. 63, no. 1, pp. 275-281, March 2017.

[7] S. I. Park et al., "Low Complexity Layered Division Multiplexing System for ATSC 3.0," IEEE Trans. Broadcast., vol. 62, no. 1, March 2016.

[8] G. Caire, G. Taricco, and E. Biglieri, "Bit-Interleaved Coded Modulation," IEEE Trans. on Information Theory, vol. 44, no. 3, pp. 927-946, May 1998.

[9] S. Ten Brink, and J. Speidel, and Ran-Hong Yan, "Iterative Demapping and Decoding for Multilevel Modulation," in 1998 IEEE Global Telecommunications Conference (GLOBECOM '98), August 1998. 
[10] C. Regueiro et al., "Improving LDPC Decoding Performance for ATSC 3.0 LDM Profiles," in 2017 IEEE International Symposium on Broadband Multimedia Systems and Broadcasting (BMSB), June 2017.

[11] R. G. Gallager, Information Theory and Reliable Communication. Wiley, 1968

[12] A. Martinez, A. G. i Fabregas, G. Caire, and F. Willems, "Bit-Interleaved Coded Modulation Revisited: A Mismatched Decoding Perspective," in 2008 IEEE International Symposium on Information Theory, July 2008, pp. 2337-2341.

[13] T. T. Nguyen and L. Lampe, "Bit-Interleaved Coded Modulation with Mismatched Decoding Metrics," IEEE Trans. on Communications, vol. 59, no. 2, pp. 437-447, February 2011.

[14] A. Alvarado, L. Szczecinski, R. Feick, and L. Ahumada, "Distribution of L-values in Gray-Mapped $M^{2}$-QAM: Closed-Form Approximations and Applications," IEEE Transactions on Communications, vol. 57, no. 7, pp. 2071-2079, July 2009.

[15] L. Michael and D. Gomez-Barquero, "Bit-Interleaved Coding and Modulation (BICM) for ATSC 3.0," IEEE Trans. Broadcast., vol. 62, no. 1, March 2016.

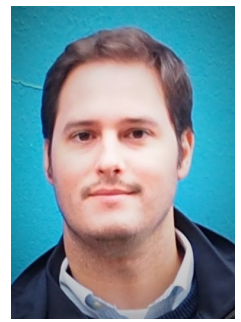

Eduardo Garro is a $R \& D$ engineer at Mobile Communications Group (MCG) of the Institute of Telecommunications and Multimedia Applications (iTEAM) at Universitat Politecnica de Valencia (UPV). He received a M.Sc. degree in Telecommunications engineering and a second M.Sc. degree in Communications and Development of Mobile Services from UPV, Spain in 2013 and 2014 respectively.

In 2012, he joined the iTEAM, and he collaborated with Agencia Nacional del Espectro (ANE) from Colombia on the coexistence between Digital Terrestrial Television (DTT) and 4G (LTE) technologies. He has also participated on the planning and optimization of DVB-T2 networks in Colombia. He has been also involved in the standardization of the new U.S. Digital Terrestrial Television (DTT) standard, ATSC 3.0.

$\mathrm{He}$ is currently pursuing his $\mathrm{Ph} . \mathrm{D}$. degree in terrestrial broadcasting and is involved in the 5G-Xcast (Broadcast \& Multicast Communication Enabler for the Fifth-Generation of Wireless Systems) project. His research activities are focused on Non-Orthogonal Multiple Access (NOMA), multiple antenna systems (MIMO), and realistic channel estimation methods in broadcasting networks.

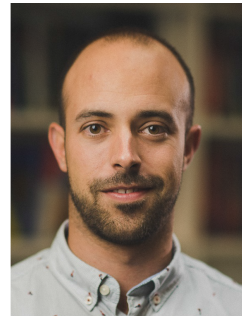

Jordi Joan Gimenez received an M.Sc. degree in Telecommunications engineering from the Universitat Politecnica de Valencia (UPV) in 2010, and a Ph.D. degree in Telecommunication from the UPV in 2015 .

$\mathrm{He}$ is a Post-Doc Researcher (Generalitat Valenciana Fellow) at iTEAM-UPV, within the research group working on terrestrial broadcasting and the optimization of next-generation broadcast systems. During his doctoral studies, he was a Guest researcher at the Royal Institute of Technology (Stockholm, Sweden), and at Teracom AB (Stockholm, Sweden), the Swedish Digital Terrestrial TV operator. He has been a Post-Doctoral Guest Researcher at the Institut für Rundfunktechnik (Munich, Germany) in 2015.

Dr. Gimenez has participated in the standardization process of the nextgeneration handheld standard DVB-NGH, as well as in the DVB technical group working on the assessment of transmission technologies for the next generation terrestrial broadcasting (DVB TM-T MIMO Study Mission). Part of the results of his research has also been presented in the ATSC 3.0 standardization process, within the Waveform Ad-Hoc group.

His main research interests include the network and frequency planning of next-generation terrestrial broadcast networks for an optimal spectrum usage. His research on the WiB (Wideband Reuse-1) concept, together with Teracom and Panasonic, was awarded with the 2016 IBC Best Conference Paper Award.

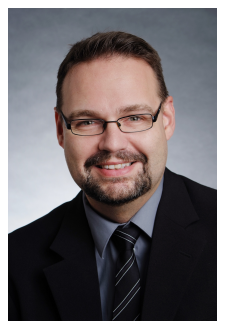

Peter Klenner received the Diploma and Ph.D. degrees in electrical engineering and communication and information technology from the University of Bremen, Germany, in 2004 and 2011, respectively.

Since 2011, he has been a Research Engineer as part of DTV Standardization with the Panasonic Langen Development Centre located close to Frankfurt, Germany. He has participated in the DVB technical group working on the assessment of transmission technologies for the next generation terrestrial broadcasting (DVB TM-T MIMO Study Mission). He has actively participated in the ATSC 3.0 standardization process. Together with Teracom and UPV, his research on the WiB (Wideband Reuse-1) concept was awarded with the 2016 IBC Best Conference Paper Award. His pursuits in research comprise, without being limited to, optimization, coding theory, iterative decoding, OFDM, MIMO communication, and massively parallel programming.

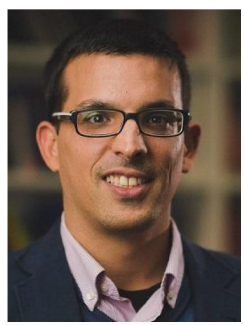

David Gomez-Barquero received the double M.Sc. degrees in telecommunications engineering from the Universitat Politecnica de Valencia (UPV), Spain, and the University of Gävle, Sweden, in 2004, the $\mathrm{Ph} . \mathrm{D}$. degree in telecommunications from the UPV in 2009; and he carried out a 2-year post-doc at the Fraunhofer Heinrich Hertz Institute, Germany.

$\mathrm{He}$ is a Senior Researcher (Ramon \& Cajal Fellow) with the Institute of Telecommunications and Multimedia Applications, UPV, where he leads a research group working on next generation broadcasting technologies. He held visiting research appointments at Ericsson Eurolab, Germany, the Royal Institute of Technology, Sweden, the University of Turku, Finland, the Technical University of Braunschweig, Germany, the Sergio Arboleda University of Bogota, Colombia, the New Jersey Institute of Technology, USA, and the Electronics and Telecommunications Research Institute, South Korea.

Dr. Gomez-Barquero has been since 2008 actively participating in the digital television standardization, including DVB-T2, T2-Lite, DVB-NGH, and ATSC 3.0. He is an Associate Editor of the IEEE TRANSACTIONS ON BROADCASTING; he edited the book Next Generation Mobile Broadcasting (CRC Press, 2013). He is the coordinator of the 5G-PPP project 5G-Xcast (Broadcast \& Multicast Communication Enablers for the Fifth-Generation of Wireless Systems). 